\title{
Diagnosis of fetal anterior abdominal wall defects by use of ultrasonography
}

BRADLEY F. SCHWARTZ, CPT, MC, USA ROBERT L. NELSON, DO

Omphalocele and gastroschisis involve protrusion of intestine through a defect in the abdominal wall. It is important to differentiate between the two because of a difference in the incidence of associated anomalies and of chromosomal abnormalities. The distinguishing characteristics are the location of umbilical cord insertion, the echogenicity of the contents of the protruding mass and, usually, the presence or absence of a sac surrounding the mass. Early detection is critical so that decisions can be made about the possibility of surgical correction and the outcome of the pregnancy. We describe an omphalocele detected by ultrasonography in the 20th week of gestation during a routine prenatal check-up.

(Key words: Omphalocele, gastroschisis, anterior abdominal wall defect, ultrasonography, pentalogy of Cantrell, Beckwith-Wiedemann syndrome)

From the Texas College of Osteopathic Medicine, Fort Worth; Dallas Fort Worth Medical Center, Grand Prairie, Tex; and Madigan Army Medical Center, Tacoma, Wash.

Presented in part at the Thirty-fourth Annual AOA Research Conference, Las Vegas, Nev, Nov 27, 1990.

The opinions or assertions contained herein are the private views of the authors and are not to be construed as official or as reflecting the views of the Department of the Army or Department of Defense.

Reprint requests to Dr Bradley F. Schwartz, CPT, MC, USA, 2d Infantry Division (Mechanized), APO San Francisco 96224 .
Omphalocele and gastroschisis are defects in the anterior part of the abdominal wall through which abdominovisceral organs protrude. The incidence of omphalocele is usually accepted as one in 5800 births, although it ranges from one in 1400 births in parts of Africa $^{1}$ to one in 10,000 births in Scandinavia. ${ }^{2}$ Gastroschisis is more uncommon, occurring in one in 12,000 live births. ${ }^{3}$

Although the causes of omphalocele are unknown, this anomaly is thought to arise from the failure of fusion of the four lateral ectomesodermic folds during the third and fourth weeks of gestation. During this time, the normal midgut and superior mesenteric artery form a physiologic hernia, protruding from the anterior aspect of the developing fetal abdomen. By the 11th week of gestation and after a 270-degree rotation, the midgut returns rapidly to the normal intra-abdominal location. The failure of this reduction subsequently results in an omphalocele. ${ }^{4}$ If the caudal or cephalic folds fail to fuse, the pentalogy of Cantrell or cloacal exstrophy of the bladder results, respectively.

The surveillance of fetal growth and development by ultrasonography usually is performed between 18 to 22 weeks' gestation. During this time, neural tube defects, genitourinary and gastrointestinal defects, cardiac anomalies, and abdominal wall defects can be detected. Furthermore, early discovery of a congenital defect will give the family ample time to contemplate the choices available to them 
concerning the outcome of the pregnancy.

When a fetal extra-abdominal mass is observed on ultrasound examination, the diagnosis of omphalocele is dependent on several structural entities:

- The location of the umbilical cord insertion in the fetus.

- The presence or absence of a sac surrounding the protruding mass, although, rarely, the sac can be absent secondary to premature rupture.

- The echogenicity of the contents of the protruding mass. The ultrasonogram demonstrates central insertion of the umbilical cord and a smooth-margined, encapsulated mass that frequently reveals homogeneous internal echoes (Table 1).

If these entities can be determined, then the correct diagnosis of omphalocele as opposed to other abdominal wall defects approaches $75 \% .^{5}$ A high degree of liver herniation and presence of fetal liver within the sac are indicators of poor prognosis for successful surgical correction.

Gastroschisis is characterized by normal cord placement with the wall defect lateral to the umbilicus, most often right-sided. The abdominal contents (mostly small intestine) will be seen free floating in the amniotic fluid with no evidence of encapsulation. Ultrasonography echoes demonstrate a lobulated margin representing the small intestine (Table 1).

These differences enable the physician to promptly and accurately diagnose an omphalocele.

\section{Report of case}

A 29-year-old gravida 2, para 0 , aborta 1 white woman was seen for fetal ultrasonography. The history indicated no congenital defects in the family nor teratogen exposure during the pregnancy. As part of this patient's evaluation, two fetal ultrasound examinations were performed, one at a level 3 neonatal center. Both examinations revealed a 20 -week intrauterine fetus of normal dimensions and movements. A homogeneous, encapsulated, spherical mass was observed protruding from the abdomen anteriorly (Figs 1 and 2). The diameter of the mass was essentially the same as that of the abdomen. The umbilical cord entered the mass centrally, with no other abdominal attachment of the cord. The mass had increased homogeneous internal echoes, having the same texture and appearance of liver tissue. Various linear fluid-filled structures within the mass were observed; the volume of internal organs in the fetal abdomen was less than is normally encountered.

A diagnosis of omphalocele was made. After thorough counseling, the family decided to terminate the pregnancy. Six days after the second ultrasound study, labor was induced and the stillborn fetus was delivered without complications.

At pathologic consultation, the following were observed: an omphalocele $5.5 \mathrm{~cm}$ in diameter characterized by a semitransparent, thin, delicate membrane containing mostly the right lobe of a large liver; cleft lip and cleft palate; mild macroglossia; cystic dilation of the ring finger on the left hand; and a short and discolored little finger on the left hand (Fig 4). Microscopically, all tissues were normal for their age and revealed no inflammation or necrosis. Chromosomal analysis of abortus tissue revealed a normal 46 , XX karyotype.

\section{Discussion}

Although not all babies with omphalocele have outcomes similar to that in the present case, certain factors indicate a poor prognosis. These indicators include coexisting physical and chromosomal anomalies (Table 2), the presence of the liver in the sac, and a large omphalocele. In contrast to gastroschisis, omphalocele is associated with an increased incidence of chromosomal abnormalities, most notably trisomies $13,15,16$, and 18 , and cardiac defects such as ventral septal defect, atrial septal defect, and tetralogy of Fallot (Table 2). In addition to these coexisting anomalies, omphalocele is associated with Beckwith-Wiedemann syndrome and the pentalogy of Cantrell. The latter syndrome, as the name implies, is associated with five anomalies: omphalocele, ectopia cordis, absent sternum, and absent portions of the diaphragm and pericardium. Beckwith-Wiedemann syndrome comprises omphalocele, macroglossia, adrenal cytomegaly, gigantism, and hyperplastic visceromegaly.

The case we report here represents the use of ultrasonography in the diagnosis of a potentially lethal birth defect during the second trimester of pregnancy. Once ultrasonography is performed and an anterior abdominal wall 


\section{MEVACOR works where it should: at the primary site of cholesterol synthesis}

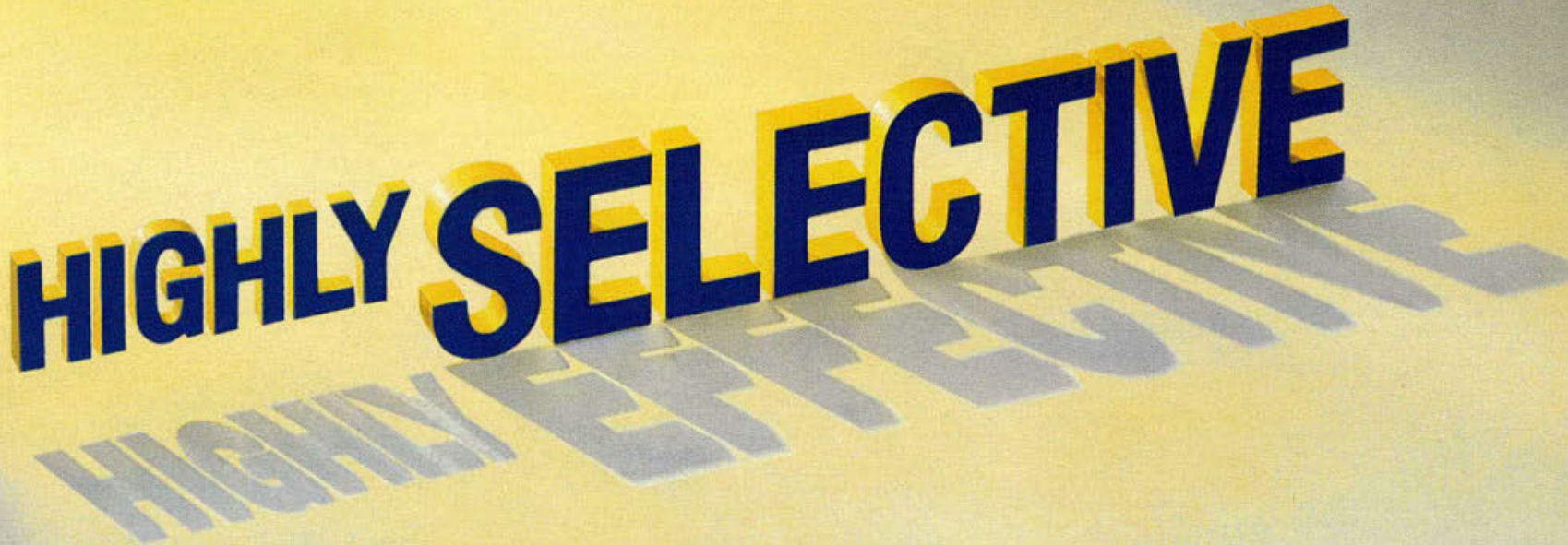

MEVACOR is the first agent to selectively inhibit cholesterol production at the primary site of synthesis. This selective activity means MEVACOR is highly effective-with only a low and variable amount of active drug available for systemic circulation.

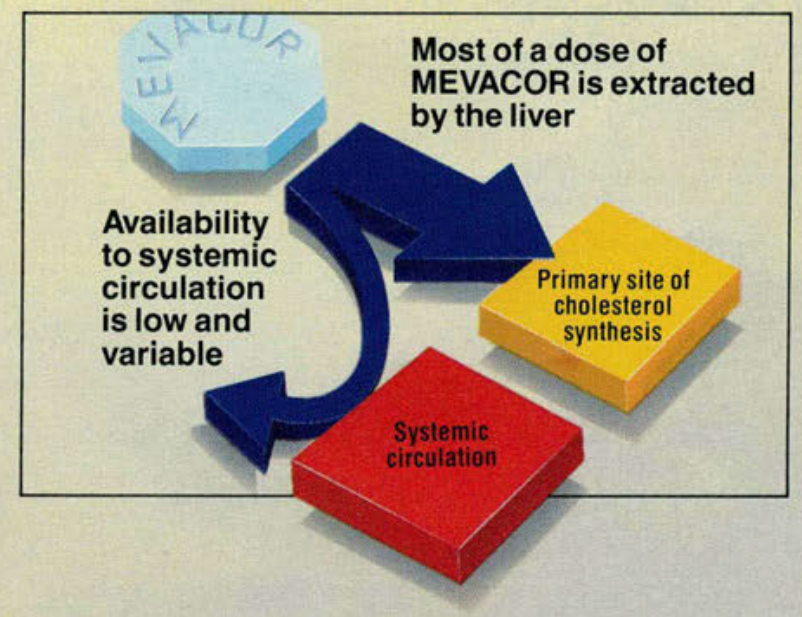

MEVACOR is contraindicated in patients who are hypersensitive to any component of the medication; in patients with active liver disease or unexplained persistent transaminase elevations; in pregnant or lactating patients; and in women of childbearing age, except when such patients are highly unlikely to conceive.

In clinical studies, marked persistent increases (to more than three times the upper limit of normal) in serum transaminases occurred in $1.9 \%$ of adult patients who received lovastatin for at least one year. It is recommended that liver function tests be performed before treatment begins, every 4 to 6 weeks during the first 15 months of therapy, and periodically thereafter in all patients.

For complete details on MEVACOR, including cautionary information regarding myopathy, drug interactions, and slit-lamp monitoring, please refer to the Prescribing Information.

For a Brief Summary of Prescribing Information, please see the back of this advertisement.

Copyright $\odot 1990$ by Merck \& Co., INC
For many patients with primary hyper-

cholesterolemia (Types Ila and IIb), when diet and other nondrug therapies are inadequate

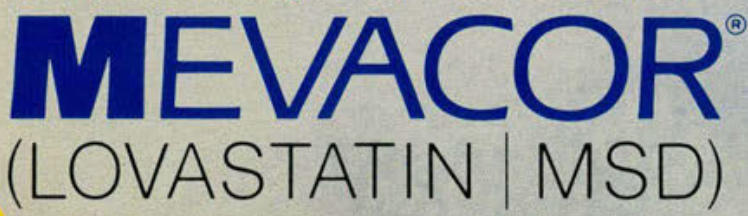

TABLETS, $20 \mathrm{mg} 40 \mathrm{mg}$ 
MEVACOR

(LOVASTATIN | MSD)

CONTRAINDICATIONS: Hypersensitivity to any component of this

Active liver disease or unexplained persistent elevations of serum Pregnancy and lactation.

Atherosclerosis is a chronic process and the discontinuation of lipidapy of primary hypercholesterolemia. Moreover, cholessential components for fetal development, including synthesis of steroids and cell membranes. Because of the ability of inhibitors of HMG-COA re ductase such as MEVACOR* (Lovastatin. MSD) to decrease the synthesis of cholesterol and possibly other products of the cholesterol biosynthesis nant woman. Therefore, lovastatin is contraindicated during pregnancy Lovastatin should be administered to women of childbearing age only pregnant while taking this drug, lovastatin should be discontinte patient should be apprised of the potential hazard to the fetus.

WARNINGS: Liver Dysfunction: Marked persistent increases (to more than 3 times the upper limit of normal) in serum transaminases occurred in $1.9 \%$ of adult patients who received lovastatin for at least usualy reil slowly to pretreatment levels. The increases usually appeare

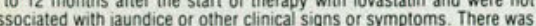
0 evidence of hypersensitivity. A liver biopsy was done in one of these eas of focal hepatitis. In this patient, transam the these patients had abnormal liver function tests prior to lovastatin therapy

It is recommended that liver function tests be performed before treatment begins, every 4106 weeks during the first 15 months of therapy with lovastatin, and periodically thereafter in all patients. Special atten tion should be paid to patients who develop elevated serum transaminase evels, and in these patients. measurements should be repeated prompty dence of progression, particularly if they rise to 3 times the upper limit of dence of progression. particularly if they rise to 3 times the upper limit of chould be considered if elevations are persistent beyond the discontinu.

The drug should be used with caution in patients who consume substantial quantities of alcohol and or have a past history of liver disease. dications to the use of lovastatin.

As with other lipid-lowering agents, moderate (less than 3 times the upper limit of normal) elevations of serum transaminases have been reported following therapy with MEVACOR (see ADVERSE REACTIONS These changes appeared soon after initiation of therapy with MEVACOA. ruption of treatment was not required.

Skeletal Muscle: Several cases of rhabdomyolysis have been associated with lovastatin therapy alone, when combined with immunosuppressive therapy including cyclosporine in cardiac transplant patients and when combined in non-transplant patients with either gemfibrozi or lipid-lowering doses ( $<1 \mathrm{~g}$ day) of nicotinic acid. Acute renal failure from rhabdomyolysis has been seen more commonly with the lovastatin-gemfibrozil combination and has also been
transplant patients receiving lovastatin plus cyclosporine.

Iransplant patients receiving lovastatin plus cyclosporine. In seriously ill patients receiving erythromycin concomitantly with
ovastatin. Therefore. patients receiving concomitant lovastatin and erythromycin should be carefully monitored.

Fulminant rhabdomyolysis has been seen as early as 3 weeks after initiation of combined therapy with gemfibrozil and lovastatin but may subjects who have had an unsatisfactory lipid response to either drug gemfibrozil do not outweigh the risks of severe myopathy, thabdomyolysis, and acute renal failure. While it is not known whether this interaction occurs with fibrates other than gemfibrozil, myopathy and rhabdomyolysis have occasionally been associated with the use of other fibrates alone, including clofibrate. Therefore, the com

of lovastatin with other fibrates should generally be avoided.
Physicians contemplating combined therapy with lovastatin and lipid-lowering doses of nicotinic acid or with immunosuppressive drugs should carefully weigh the potential benefits and risks and should carefully monitor patients for any signs and symptoms of muscle pain. apy and during any periods of upward dosage titration of either drug. Periodic CPK determinations may be considered in such situations, but there is no assurance that such monitoring will prevent the occurrence of severe myopathy. The monitoring of lovastatin drug and metabolite levels may be considered in transplant patients who are treated with mmunosuppressives and lovastatin.

Lovastatin therapy should be temporarily withheld or discontinued in any patient with an acute, serious condition suggestive of a myopathy or
having a risk factor predisposing to the development of renal failure secondary to rhabdomyolysis, including severe acute infection, hypo. lension, major surgery, trauma, severe metabol

Myalgia has been associated with lovastatin therapy. Transient, mildly treated creatine phosphokinase levels are commonly seen in lovastatin. patients developed a myopathy, i.e., myalgia or muscle weakness sidered in any patient with diffuse myalgias. muscle tenderness, or weak ness, and of marked elevation of CPK. Patients should be advised report promptly unexplained muscle pain, tenderness. or weakness. pardiscontinued it markedly elevated CPK levels occur or myopathy is diag.

Most of the patients who have developed myopathy (including rhabdo-
myolysis) while taking immunosuppressive drugs, gemfibrozil, or lipid-lowering doses of nicoimmunosuppressive therapy including cyclosporine developed myop. athy: the corresponding percentages for gemfibrozil and niacin were In 6 patients with cardiac transplants taking immunosuppressive therapy including cyclosporine concomitantly with lovastatin $20 \mathrm{mg} /$ day, the
average plasma level of active metabolites derived from lovastatin was average plasma level of active metabolites derived from lovastatin was elevated to approximately 4 times the expected leveis. Because of an apderived from lovastatin and myopathy, the daily dosage in patients taking ADMiNISTRATION). Even at this dosage, the benefits and risks of using lovastatin in patients taking immunosuppressants should be carefully
RECAUTIONS: General: Before instituting therapy with MEVACOR-
ovastatin, MSO), an attempt should be made to control hypercholesterand to treat other underlying medical problems (see INDICATIONS AND Lovastatin may elevate creatine phosphokinase and transaminase lev-
els (see ADVERSE REACTIONS). This should be considered in the differ-
ential diagnosis of chest pain in a patient on therapy with lovastatin. Eye: There was a high prevalence of baseline lenticular opacities in the
patient population included in the clinical trials with lovastatin. During patient population included in the clinical trials with lovastatin. During
these trials the appearance of new opacities was noted. The causal rela Of 431 patients examined with slit lamp at baseline and during with lovastatin. 34 had opacities reported at the final examination ( 5 to 15 months after starting lovastatin) that were not noted at baseline. On the

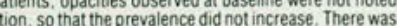
opacities reported. nor was any patient, including those with opacities voted at baseline, discontinued from therapy because of a decrease in visual acuity. Nevertheless, until further experience is obtained. it is rec-
ommended that patients placed on lovastatin therapy be examined with a slit lamp

Homozygous familial Hypercholesterolemia: MEVACOR is less effective in patients with the rare homozygous familial hypercholesterol-
emia. possibly because these patients have no functional $L \mathrm{~L}$ receptors. MEVACOR appears to be more likely to raise serum transaminases (see ADVERSE REACTIONS) in these homozygous patients.

Drug Interactions: Immunosuppressive Drugs, Gemfibrozil, Niacin
(Nicotinic Acid). Erythromycin: See WARNINGS. Skeletal Muscle. Coumarin Anticoagulants: In a clinical trial in wartarin-treated patients designed specifically to observe a potential effect of lovastatin on the proany consistent alteration of the anticoagulant action of wartarin. However. since the drug was marketed, clinically evident bleeding and or increased
prothrombin time have been reported in a tew patients taking coumarin prothrombin time have been reported in a few patients taking coumarin
anticoagulants concomitantly with lovastatin. The causal relationship to lovastatin is unclear. Nevertheiess, it is recommended that in patients taring anticoagulants, prothrombin time be determined before starting lo significant alteration of prothrombin time occurs. Once a stable prothrom.
bin time has been documented. prothrombin times can be monitored at the intervals usually recommended for patients on coumarin anticoagulants. If the dose of lovastatin is changed, the same procedure should be
repeated. Lovastatin therapy has not been associated with bleeding or with changes in prothrombin time in patients not taking anticoagulants
Antipyrine: Antipyrine is a model for drugs metabolized by the micro somal hepatic enzyme system (cytochrome P450 system). Because lov astatin had no effect on the pharmacokinetics of antipyrine, interaction
with other drugs metabolized via this mechanism are not expected.

with other drugs metabolized via this mechanism are not expected.
Propranolol. In normal volunteers. there was no clinically significant
pharmacokinetic or pharmacodynamic interaction with concomitant pharmacokinetic or pharmacodynamic interaction with
administration of single doses of lovastatin and propranolo Digoxin: In patients with hypercholesterolemia, concomitant adminis-
tration of lovastatin and digoxin resulted in no effect on digoxin plasma Other Concomitant Therapy: Although specific interaction studies were not performed, in clinical studies, lovastatin was used concomitantly with beta blockers, calcium channel blockers, diuretics, and nonsteroidal anti-
inflammatory drugs (NSAIDs) without evidence of clinically significant adverse interactions.

Carcinogenesis, Mutagenesis, Impairment of Fertility: In a 21-month the incidence of hepatocellular carcinomas and adenomas was observed at doses of $500 \mathrm{mg} / \mathrm{kg}$ day ( 312 times the maximum recommended doses of 20 and $100 \mathrm{mg} / \mathrm{kg} /$ day ( 12.5 and 62.5 times the maximum rec-

A statistically significant increase $(p \leq 0.05)$ in the incidence of pulmo-
nary adenomas was seen in female mice receiving $500 \mathrm{mg} / \mathrm{kg}$ day ( 312 times the maximum recommended human dose); no similar changes were seen in males at any dose or in
dayse the incidence of pulmonary tumors was within the range of
cause cause the incidence of pulmonary tumors was within the range of
untreated animals in studies of similar duration. the relationship of this atter change to treatment is not known.

In addition, an increase in the incidence of papilloma in the non-glandu-
ar mucosa of the stomach was observed in mice receiving 100 and 500 $\mathrm{mg} / \mathrm{kg}$ day $(62.5$ and 312 times the maximum recommended human maximum recommended human dose). The glandular mucosa was not affected. The human stomach contains only glandular mucosa. Impor.
tantly, there is a strong association between this change and hyperplasia of the squamous epithelium (acanthosis) in this region; acanthosis is a characteristic change observed in the non-glandular mucosa of rodents
treated with HMG.COA reductase inhibitors and is most probably a result of inhibition of the reductase in this tissue.
Similar squamous epithelium is found in the esophagus and anorectal junction of the mouse and rat; however, no evidence of a similar drug: induced hyperplastic response was observed in these tissues in studies of up to 21 months in the mouse given up to $500 \mathrm{mg} / \mathrm{kg}$ day (312 times the
maximum recommended human dose), or in a study of 24 months in the In a 24-month carcinogenicity study in rats, there was a positive dose response relationship for hepatocellular carcinogenicity in males (unad-
usted $p=0.025$. However, because the incidence of hepatocellular carjusted $p=0.025$ ). However, because the incidence of hepatocellular car-
cinogenicity observed in male rats in this study is similar to that observed unclear.

No evidence of mutagenicity was observed in a microbial mutagen test using mutant strains of Salmonella typhimurium with or without rat or genetic material was noted in an in vitro alkaline elution assay using rat or vitro chromosome aberration study in CHO cells, or an in vivo chromoNo drug-related effects on fertility were found in studies with rats. Pregnancy: Pregnancy Category $X$ : See CONTRAINDICATIONS Lovastatin has been shown to produce skeletal maliormations in the rat Ltus at doses of $800 \mathrm{mg} / \mathrm{kg}$ day ( 500 times the maximum recommended thens was observed. These individual changes are within the range of changes were seen in either species at doses of up $1080 \mathrm{mg} / \mathrm{kg}$ day ( 50 mations was noted in rabbits at up to $15 \mathrm{mg} \mathrm{kg}$ day (highest tolerated dose about 9 times the maximum recommended human dose). There are no ata in pregnant women.

Nursing Mothers: Studies in rats have shown that lovastatin is excreted in Because many drugs are excreted in human milk and because of the women taking lovastatin should not nurse their infants (see
CONTRAINDICATIONS).

Pediatric Use: Safety and effectiveness in children have not been estabfor at least a decade and because experience with this drug is limited (no
DVERSE REACTIONS: MEVACOR» (Lovastatin, MSD) is generally well tolerated; adverse reactions usually have been mild and transient. Less to adverse experiences attributable to MEVACOR. About $2 \%$ of patients adverse experiences attributable to MEVACOR: about one-third of these

Clinical Adverse Experiences: Adverse experiences reported in patients
treated with MEVACOR in controlled clinical studies are shown in the table below:

$\begin{array}{lll}\text { MEVACOR } & \text { Placebo } & \text { Cholestyrt } \\ (\mathrm{N}=613) & (\mathrm{N}=82) & (\mathrm{N}=88)\end{array}$

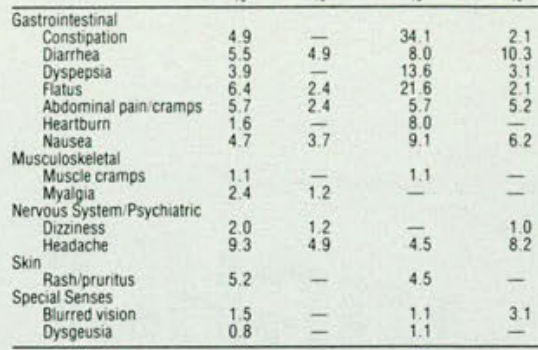

Laboratory Tests: Marked persistent increases of serum transaminases ve been noted (see WARNINGS)

About $11 \%$ of patients had elevations of creatine phosphokinase (CPK)
evels of at least twice the normal value on one or more occasions. The probucol. $2 \%$. This was attributable to the noncardiac fraction of CPK
arge increases in CPK have sometimes been reported (see WARNINGS, arge increases in
Skeletal Muscle

Concomitant Therapy: In controlled clinical studies in which lovastatin was administered concomitantly with cholestyramine, no adverse reacreactions that occurred were limited to those reported previousily with lovastatin or cholestyramine. Other lipid-lowering agents were not admin uncontrolled clinical studies. most of the patients who have developed myopathy were receiving concomitant therapy with immunosuppressive
drugs. gemfibrozil, or niacin (nicotinic acid) (see WARNINGS. Skeleta

Uncontrolled Clinical Studies: The adverse experiences observed in uncontrolled studies were similar to those seen in controlled clinical
studies. Abnormal liver function tests were observed at a higher incidence athy (myalgia with marked CPK elevations) was reported in approx

Causal Relationship Unclear: Nervous System: Peripheral neuropathy has been reported; the relationship to lovastatin is uncertain. Visua phy in over 30 patients showed no evidence of neurotoxic effects of Specia/ Senses: Of 431 patients examined with slit lamp at baseline an ination ( 5 to 15 months atter starting lovastatin) that were not noted at were not noted at the final examination, so that the prevalence did not increase (see PRECAUTIONS)

Post-marketing Experience: Additional adverse experiences occurring Proe the drug was marketed are listed below

omiting. has been reported rarely which has included one or more of the following
features: anaphylaxis, angicederna, lupus-like syndrome, polymyalgia ANeumatica, thrombocytopenia, leukopenia. hemolytic anemia, positiv ivity, fever, flushing, malaise, and dyspnea.
Nervous SystemPsychiatric: Psychic disturbances, including Skin: Erythema multiform
drome: toxic epidermal necrolysis.

Causal Relationship Unknown

Gastrointestinal: Pancreatitis, stomatitis.

Nervous System Psychiatric: Depression, insomnia.
Metabolic: Edema.

Linical Laboratory Test Findings

Liver function fests: Liver function test abnormalities, including
elevated alkaline phosphatase and bilirubin. Thyroid Function fests: Rare reports of thyroid function test abno

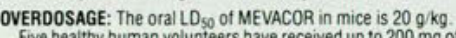

Five healthy human volunteers have received up to $200 \mathrm{mg}$ of lovastatin as a single dose without clinically significant adverse experiences. A few specific symptoms, and all paten
imum dose taken was 5 to 6

Until further experience is obtained, no
age with MEVACOR can be recommended.

The

DOSAGE AND ADMINISTRATION: The patient should be placed on a standard cholesterol-lowering diet before receiving MEVACOR and should The recommended starting dose is $20 \mathrm{mg}$ once a day given with the evening meal. The recommended dosing range is $201080 \mathrm{mg}$ day in sin. Adjustments of dosage should be made at intervals of 4 weeks or more.
Doses should be individualized according to the patient's response (see Tables I to IV under CLINICAL PHABMACOLOGY Cinica Siudes tor dose
response results). or those patients with severely elevated serum cholesterol levels
$>300 \mathrm{mg} / \mathrm{dL}$ in patients taking immunosuppressive drugs concomitantly with mended dosage is $20 \mathrm{mg}$ day.

MSD and consideration should be given to reducing the tosage of MEV

DOHME

For more detailed information, consult your MSD Representative or see Prescribing Information, Merck Sharp \& Dohme, Division of Merck \& Co., IMC., West Point, PA 19486. 
Table 1

Ultrasonic and Gross Characteristics of Gastroschisis and Omphalocele

\begin{tabular}{|lll|}
\hline Characteristic & \multicolumn{1}{c|}{ Gastroschisis } & Ompalocele \\
\hline $\begin{array}{l}\text { - Umbilical cord } \\
\text { Location }\end{array}$ & Lateral to the defect & Central in the sac \\
Insertion point & Directly into abdomen & Protruding sac \\
- Encapsulation & Absent & Present \\
- Echogenicity & Lobulated with mixed internal echoes & Homogeneous internal echoes \\
\hline
\end{tabular}

defect is observed, it is vital that the diagnosis be made with speed and accuracy. The differential diagnosis for abdominal wall defects includes both omphalocele and gastroschisis. The differences between the two are distinct. $6-8$ The ultrasonographic differences are shown in Table 1 and Figures 1 through 3 . An omphalocele is covered by a translucent membrane that is composed of an internal peritoneum and an external amnion. These two layers are separated by a thin layer of Wharton's jelly. The protruding sac can contain intestine, bladder, liver, stomach, or spleen (or a combination). The umbilical cord attaches to the apex of this mass and finds no other insertion in the fetus.

In constrast to omphalocele, gastroschisis has no sac surrounding the protruding abdominal contents. This leaves the extra-abdominal viscera, most often small intestine, to float freely in the amniotic fluid. The umbilical cord is attached lateral to the abdominal wall defect, most often to the left of the defect. In gastroschisis, this defect is usually much smaller. Associated anomalies are much less common and are mainly confined to the jejunoileal region in the forms of nonrotation, malrotation, and atresia of the bowel.

Some studies found that gastroschisis is caused by a teratogenic insult to the mother
Table 2

Congenital Anomalies Associated With Omphalocele

Common anomalies

Trisomies 13, 15, 16, 18

Ventral septal defect

Atrial septal defect

Tetralogy of Fallot

Polycystic kidney

Renal agenesis

Anencephaly

Hydrocephaly

Microcephaly

Cleft lip

Cleft palate

Malrotation of the gut during pregnancy. ${ }^{9}$ It is also suggested that omphaloceles are the result of natural inborn errors, and possibly genetic aberrations. Because of the associated anomalies with omphalocele, there is some indication of genetic origin of the disorder. ${ }^{10,11}$ Many studies ${ }^{10-14}$ show a genetic predisposition for the etiology of omphalocele. One study ${ }^{12}$ supports sex-linked recessive inheritance. An African study ${ }^{1}$ reported that the incidence of omphalocele was approximately 1 in 1400 births in the country of Nigeria.

In contrast, other studies indicate no genetic predisposition greater than that of the general population. Studies from British Columbia and the Hungarian Register have indicated that the risk of having recurrent omphaloceles 
Figure 1. Coronal view ultrasonogram at 20 weeks' gestation. Notice the smooth, encapsulated protruding omphalocele (o).

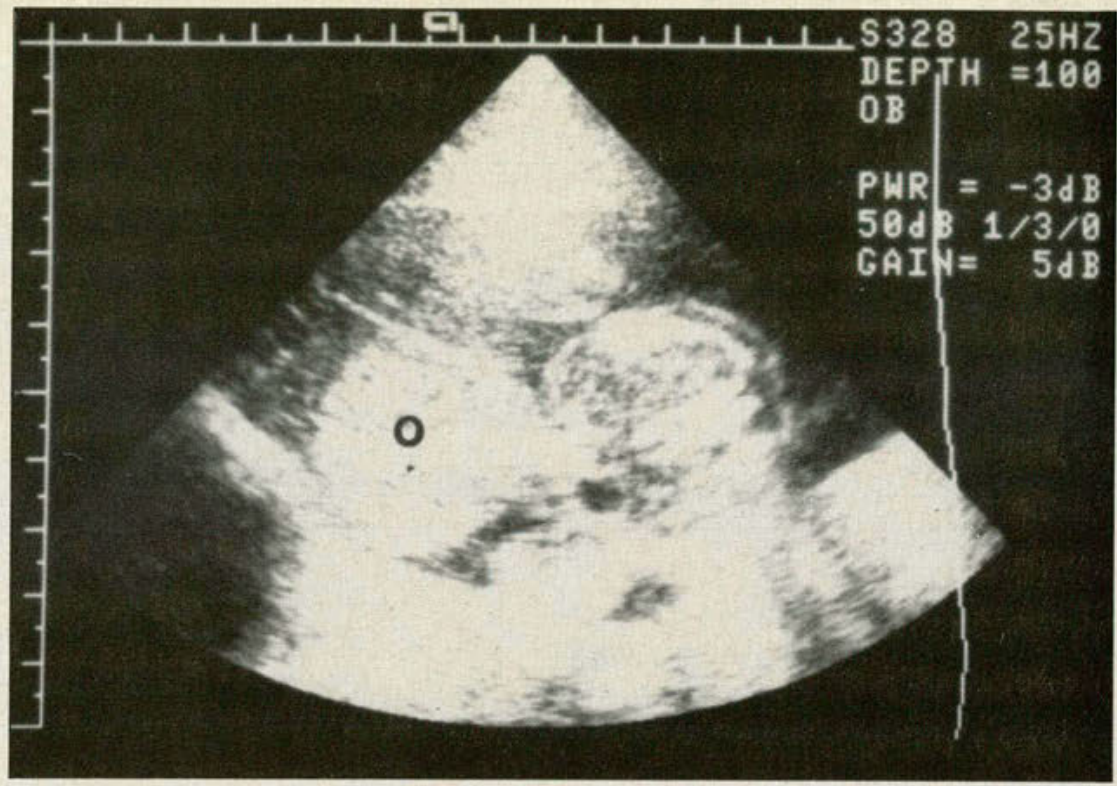

Figure 2. Parasagittal view ultrasonogram of same patient. $0=$ omphalocele; $b=$ body of the fetus.

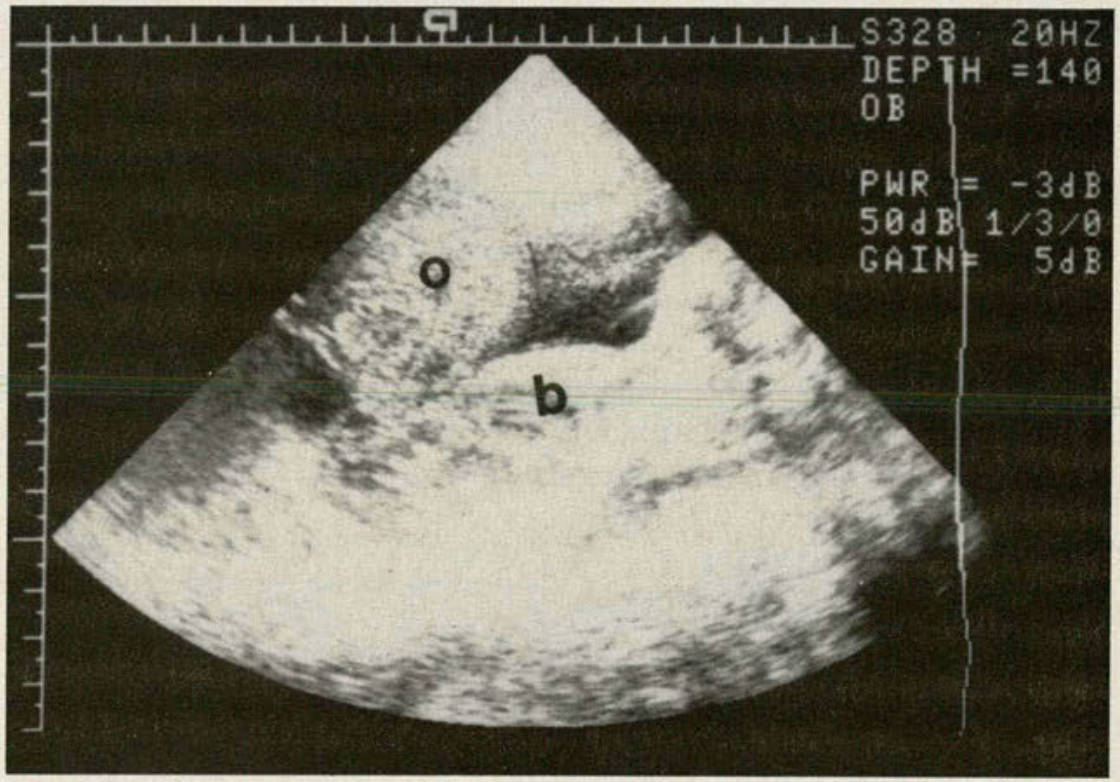

within a family remains no higher than that of the general population. ${ }^{15,16}$ Possibly the reason for the few studies indicating a familial occurrence, that is the infrequency with which familial cases occur, makes them popular research subjects.

\section{Conclusion}

Careful surveillance and diligence in the diagnosis of this defect in utero is vital if the appropriate choices are to be made in the outcome of the pregnancy. Only after careful searching by means of fetal ultrasonography can the family make such important decisions. The family physician must be knowledgeable about the capabilities and limitations of the diagnostic tools available. The family physician must be familiar with tertiary care centers and not hesitate to refer patients to specialized care in this field.

The case reported here in no way implies that termination is the only alternative to a pregnancy complicated by omphalocele. Both omphalocele and gastroschisis are amenable 

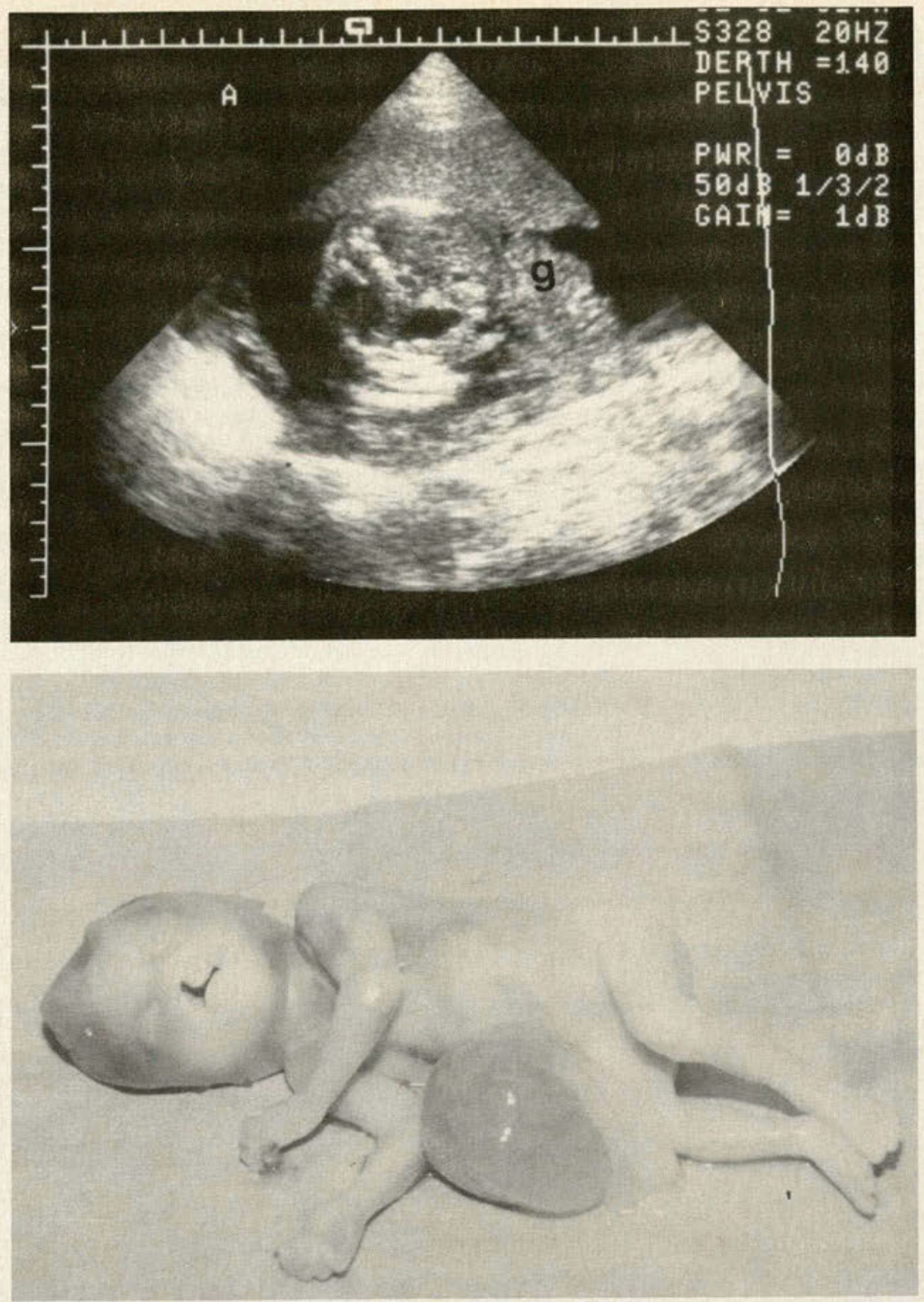

Figure 3. Coronal view of a 20-week fetus with gastroschisis. Notice the freefloating small intestines in the amniotic sac, (g).

Figure 4. Gross specimen of stillborn 20-week fetus. Notice the prominent omphalocele, macroglossia, cleft lip and palate, and the cyst on left ring finger.

to corrective surgery. The outcome of the surgery has varying success based on several prognostic indicators, including those mentioned earlier in this report. ${ }^{17}$ Furthermore, karyotyping by means of umbilical blood sampling, transabdominal chorionic villi sampling, or amniocentesis should be performed to rule out any chromosomal abnormalities. In addition, careful fetal ultrasonography should be done, and repeated if necessary, to search for those anomalies (Table 2) and other syndromes most closely associated with omphalocele. If karyotyping and ultrasound at a tertiary care center reveal no other gross abnormalities, pediatric surgical correction can be extremely successful. Because of the possible relationship to chromosomal inheritance, genetic counseling of affected women who wish to have more children is warranted.

The case described here is an example of a potentially life-threatening birth defect that requires rapid, but very careful, prenatal diagnosis. Such diagnosis is best handled by a team of healthcare personnel that includes geneti- 
cists, radiologists, neonatologists, pediatricians, pathologists, and obstetricians.

The authors wish to acknowledge the financial and medical contributions of Frederick Wilkins, DO, David Barker, PhD, and Ray Page, PhD, of the Texas College of Osteopathic Medicine, Fort Worth.

1. Gupta B: Incidence of congenital malformations in Nigerian children. West Afr Med J 1969;18:22-27.

2. Lindham S: Omphalocele and gastroschisis in Sweden, 19651976. Acta Paediatr Scand 1981;70:55-60.

3. Baird PA, MacDonald EC: An epidemiologic study of congenital malformations of the anterior abdominal wall in more than half a million consecutive live births. Am J Hum Genet 1981;33:470-478.

4. Moore KL: The Developing Human, ed 4. WB Saunders Co, 1988, p 231.

5. Bair JH, Russ PD, Pretorius DH, et al: Fetal omphalocele and gastroschisis: A review of 24 cases. AJR 1986;147:10471051.

6. Moore TC: Gastroschisis and omphalocele: Clinical differences. Surgery 1977;82:561-568.
7. Noordijk JA, Bloemsma-Jonkman F: Gastroschisis: No myth. $J$ Pediatr Surg 1978;13:47-49.

8. Osborne J: Gastroschisis and omphalocele: Prenatal ultrasonic detection and its significance. Australas Radiol 1986;30(2):113-116.

9. Duhamel B: Embryology of exomphalos and allied malformations Arch Dis Child 1963;38:142-147.

10. DiLiberti JH: Familial omphalocele: Analysis of risk factors and case report. Am J Med Genet 1982;13:263-268.

11. Adeyokunnu AA, Akingbehin NA: Experience with the management of exomphalos and gastroschisis in Ibadan. Nig $J$ Paediat 1981;8:45-51.

12. Havalad S, Noblett H, Speidel BD: Familial occurrence of omphalocele suggesting sex-linked inheritance. Arch Dis Child 1979;54:142-151.

13. Kapur S, Higgins JV, Scott-Emuakpor AB, et al: Omphalocele in half-siblings. Clin Genet 1980;18:88-90.

14. Lurie IW, Ilyina HG: Familial omphalocele and recurrence risk. Am J Med Genet 1984;17:541-543.

15. Lowry RB, Baird PA: Familial gastroschisis and omphalocele. Am J Hum Genet 1982;34:517-518.

16. Czeizel A: Recurrence risk of omphalocele, letter. Lancet $1979 ; 2: 470$

17. Yaster M, Scherer TL, Stone MM, et al: Prediction of surgical primary closure of congenital abdominal wall defects using intraoperative measurements $J$ Pediatr Surg 1989;24:12171220 . 